\title{
High-gain Frequency Reconfigurable Vivaldi Antenna
}

\author{
C. Borda Fortuny* ${ }^{* \dagger}$, K. F. Tong* ${ }^{*}$ K. Chetty ${ }^{\dagger}$ \\ ${ }^{*}$ Department of Electronic and Electrical Engineering \\ ${ }^{\dagger}$ Department of Security and Crime Science \\ University College London (UCL) \\ London, UK
}

\author{
D. M. Benton \\ L-3 TRL Technology \\ Tewkesbury, Gloucestershire, UK
}

\begin{abstract}
This article proposes a frequency agile antenna whose operating frequency band can be switched. The design is based on a Vivaldi antenna. High-performance radio-frequency microelectromechanical system (RF-MEMS) switches are used to realize the $2.7 \mathrm{GHz}$ and $3.9 \mathrm{GHz}$ band switching. The low band starts from $2.33 \mathrm{GHz}$ and works until $3.02 \mathrm{GHz}$ and the high band ranges from $3.29 \mathrm{GHz}$ up to $4.58 \mathrm{GHz}$. The average gains of the antenna at the low and high bands are 10.9 and $12.5 \mathrm{dBi}$, respectively. This high-gain frequency reconfigurable antenna could replace several narrowband antennas for reducing costs and space to support multiple communication systems, while maintaining good performance.
\end{abstract}

\section{INTRODUCTION}

The growth of reliable wireless communications demands higher antenna capabilities when compared to common antenna designs. New requirements for increased functionality encompass, but are not limited to, compact size, low cost, high efficiency and wide bandwidth with stable radiation patterns [1]. Multiband antennas supporting different frequency bands may not be a suitable solution, in terms of maintaining the highest signal to noise ratio (SNR) and efficiency [2]. Frequency reconfigurable antennas are an attractive and feasible solution, because they can operate in one frequency band while rejecting the others, thereby increasing SNR. Some applications for reconfigurable antennas are cognitive radio and multiple-input-multiple-output (MIMO) systems [3].

Vivaldi antennas are frequency-independent antennas, which permits high directivity and stable beamwidth in a wide range of frequencies [4]. Some work on reconfigurable Vivaldi antennas has been reported in [5]-[7]. In [5], two rings are introduced to a Vivaldi antenna to obtain narrowband reconfiguration, however the gain that can be achieved is low, only up to $5.93 \mathrm{dBi}$. In [6], several ring resonators are introduced on the tapered slot to achieve one wide band and three narrow bands. The operating frequency can be switched between $1.10 \mathrm{GHz}, 2.25 \mathrm{GHz}$ and $3.10 \mathrm{GHz}$. However, the measured gain of the antenna is $3.27 \mathrm{dBi}$ in the wideband mode and less than $1.6 \mathrm{dBi}$ in the narrow bands. Thus, the radiation beams are broad, making the antenna almost omnidirectional in the narrowband modes. Finally, in [7] ring resonators are used to switch between narrow bands, but, again, it displays low gain.

The design proposed here is a high-gain antenna that can be switched between two frequency bands, centered at
2.7 $\mathrm{GHz}$ and $3.9 \mathrm{GHz}$. In order to improve the SNR of the antenna, a quarter-wavelength microstrip stub is used to control the operating frequency. Given that the Vivaldi antenna is frequency-independent, the radiation characteristics are going to be maintained in its whole band. The antenna is electronically reconfigurable by using two RF-MEMS switches which change the length of the current path for different bands.

\section{Antenna Geometry And Design}

The antenna is designed on a single microwave substrate, as shown in Fig. 1. The top layer is composed of a slot inserted in an aperture, making the stable beamwidth possible from 1 to $7 \mathrm{GHz}$. The dielectric layer is Taconic RF-43, which is a low loss alternative to FR-4. The thickness of the substrate is $0.79 \mathrm{~mm}$ with a dielectric constant, $\varepsilon_{r}$, of 4.3. A dielectric extension has been added at the end of the aperture in a semicircular shape, becoming a better interface between air and the antenna. In the bottom layer, a microstrip line is used to feed the antenna. The position and length of the microstrip line and the widths of the microstrip and slot line have been optimized for maximizing the signal coupling.

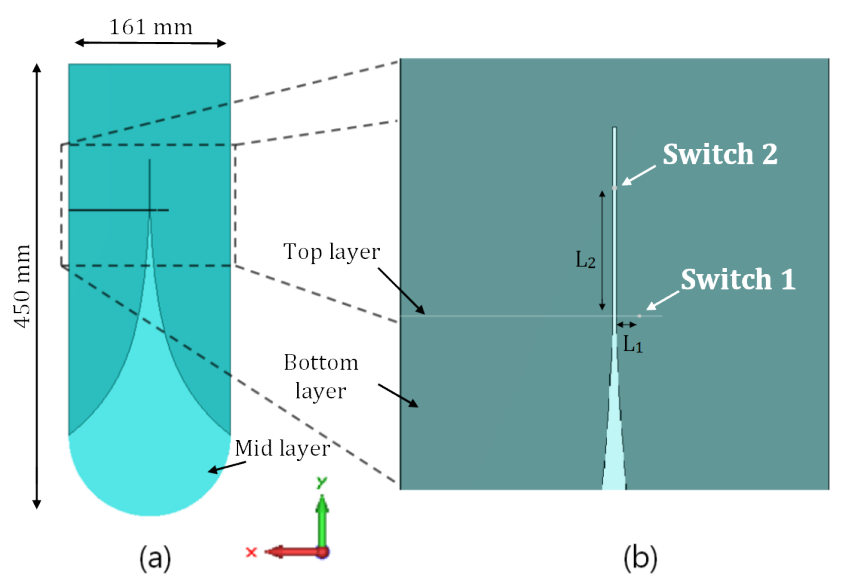

Fig. 1. The dual-band reconfigurable Vivaldi antenna geometry (a) whole view (b) arrangement of the switches

The two switches used to reconfigure the antenna are RMSW100HP from Radant MEMS. This RF-MEMS switches 
can work from DC to $12 \mathrm{GHz}$, with maximum $0.20 \mathrm{~dB}$ insertion loss and up to $20 \mathrm{~dB}$ isolation in the bands of interest. The frequency response of the switches has been analysed and considered in the antenna simulation using CST Microwave Studio [8]. The position of the switches is shown in Fig. 1](b). These locations has been optimized for switching between high band and low band, so that they shorten the current path while setting a maximum in the microstrip-to-slotline junction when operating at the high band. The distance to the junction is calculated as shown in (1), where $\lambda_{g, H B}$ is the guided wavelength in the microstrip line and $\lambda_{o, H B}$ is the freespace wavelength, at the center frequency of the high band. The operation of the switches for the two bands is shown in Table [I

$$
L_{1}=\frac{\lambda_{g, H B}}{4} \quad, \quad L_{2}=\frac{3}{4} \cdot \lambda_{o, H B}
$$

TABLE I

OPERATION OF THE SWITCHES FOR THE TWO FREQUENCY BANDS.

\begin{tabular}{|c|c|c|}
\hline Frequency band & Switch 1 (microstrip) & Switch 2 (slotline) \\
\hline Low band & ON & OFF \\
\hline High band & OFF & ON \\
\hline
\end{tabular}

\section{RESULTS AND DISCUSSION}

The simulated $S_{11}$ parameter and gain at $(\theta, \phi)=$ $\left(-90^{\circ}, 90^{\circ}\right)$ of the antenna operating in the two modes are shown in Fig. 2. The gains for each band are stable within the bands and drop rapidly in the immediate proximity of the two ends of the band. Table $\Pi$ presents the results obtained.

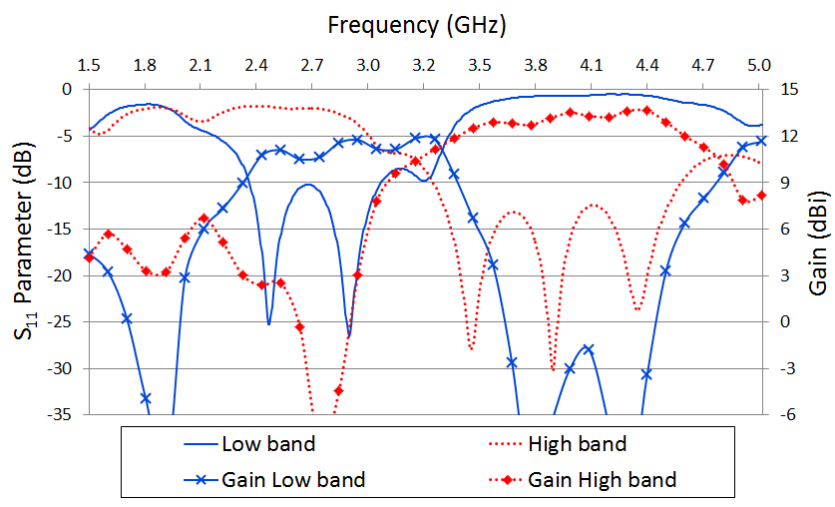

Fig. 2. Simulated $S_{11}$ parameter and realized gain of the proposed design.

TABLE II

SIMULATED RESULTS FOR THE PROPOSED ANTENNA.

\begin{tabular}{|c|c|c|c|}
\hline & Frequency Range (Center) & Bandwidth & Gain \\
\hline Low band & $2.33-3.02 \mathrm{GHz}(2.7 \mathrm{GHz})$ & $0.69 \mathrm{GHz}$ & $10.0-11.78 \mathrm{dBi}$ \\
\hline High band & $3.29-4.58 \mathrm{GHz}(3.9 \mathrm{GHz})$ & $1.29 \mathrm{GHz}$ & $11.3-13.65 \mathrm{dBi}$ \\
\hline
\end{tabular}

The normalized radiation patterns of the proposed antenna are shown in Fig. 3. Half-Power Beamwidth (HPBW) of the
E-plane is about $33.9^{\circ}$ for the low band and $29.9^{\circ}$ for the high band. The HPBW of the H-plane for the low band is $52.3^{\circ}$ and for the high band $41.4^{\circ}$. Therefore, the radiation pattern for both bands is similar in E and H-plane.

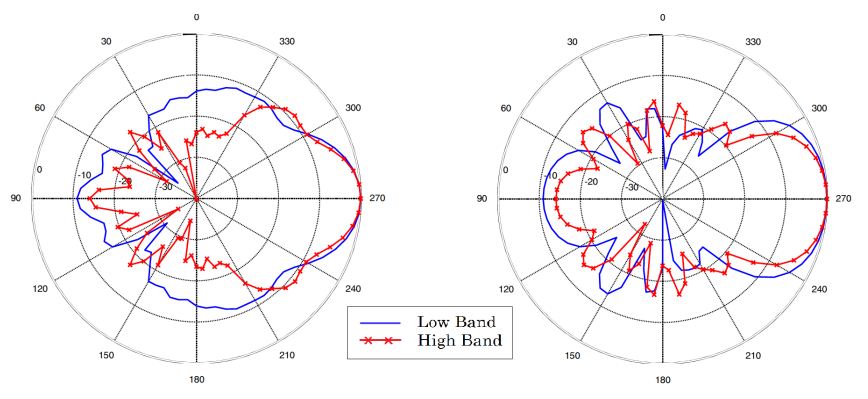

Fig. 3. Simulated E-plane radiation pattern (left) and H-plane radiation pattern (right) of the proposed design.

\section{CONCLUSION}

A Vivaldi antenna which can operate in two adjacent bands has been presented. The two bands are 2.33 to $3.02 \mathrm{GHz}$ and 3.29 to $4.58 \mathrm{GHz}$ and there is at least $7 \mathrm{~dB}$ isolation between them. A stable gain is achieved, around $10.9 \mathrm{dBi}$ in the low band and $12.5 \mathrm{dBi}$ in the high band.This low cost and compact design can support existing multiple wireless communication systems.

Surface mounted RF-MEMS are used to redistribute current paths in the antenna because they are easy to integrate into the antenna structure. Although they have some drawbacks, e.g. insertion losses, this design minimizes their effects because only one switch is working at a time.

The proposed antenna is currently being fabricated and will be tested to verify the simulation results. The measured results will be presented in the conference.

\section{ACKNOWLEDGEMENT}

This work is jointly funded by EPSRC UK, grant reference number: EP/G037264/1, and L-3 TRL Technology.

\section{REFERENCES}

[1] J. T. Bernhard, "Reconfigurable antennas and apertures: state of the art and future outlook," pp. 1-9, 2003. [Online]. Available: http://dx.doi.org/10.1117/12.497433

[2] D. Peroulis, K. Sarabandi, and L. Katehi, "Design of reconfigurable slot antennas," Antennas and Propagation, IEEE Transactions on, vol. 53, no. 2, pp. 645-654, 2005 .

[3] C. Christodoulou, Y. Tawk, S. Lane, and S. Erwin, "Reconfigurable antennas for wireless and space applications," Proceedings of the IEEE, vol. 100 , no. 7, pp. 2250-2261, 2012.

[4] P. J. Gibson, "The vivaldi aerial," in Microwave Conference, 1979. 9th European, 1979, pp. 101-105.

[5] M. Hamid, P. Gardner, P. Hall, and F. Ghanem, "Multimode vivaldi antenna," Electronics Letters, vol. 46, no. 21, pp. 1424-1425, 2010.

[6] M. Hamid, P. Hall, P. Gardner, and F. Ghanem, "Frequency reconfigurable vivaldi antenna," in Antennas and Propagation (EuCAP), 2010 Proceedings of the Fourth European Conference on, 2010, pp. 1-4.

[7] M. Hamid, P. Gardner, P. Hall, and F. Ghanem, "Switched-band vivaldi antenna," Antennas and Propagation, IEEE Transactions on, vol. 59, no. 5, pp. 1472-1480, 2011.

[8] "CST Microwave Studio." [Online]. Available: https://www.cst.com/ Products/CSTMWS/ 